\title{
Impact of posttraumatic stress disorder on sinonasal symptoms and quality of life in patients with chronic rhinosinusitis
}

This article was published in the following Dove Press journal:

Patient Preference and Adherence

29 November 2012

Number of times this article has been viewed

\author{
Dražen Shejbal' \\ Davor Vagić ${ }^{2}$ \\ Siniša Stevanović ${ }^{3}$ \\ Elvira Koić ${ }^{4}$ \\ Livije Kalogjera ${ }^{2}$ \\ 'Division of Otorhinolaryngology, \\ Pakrac City Hospital, Pakrac, Croatia; \\ ${ }^{2}$ Department of Otorhinolaryngology \\ and Head and Neck Surgery, Sisters \\ of Mercy Hospital, Zagreb, Croatia; \\ ${ }^{3}$ Division of Otorhinolaryngology, \\ ${ }^{4}$ Division of Psychiatry, Virovitica \\ General Hospital, Virovitica, Croatia
}

Correspondence: Dražen Shejbal Pakrac City Hospital, Bolnička 68, 34550 Pakrac, Croatia

Tel +3859l। 515929

$\mathrm{Fax}+38543333091$

Email dr.azen@vip.hr
Background: Severity of chronic rhinosinusitis (CRS), measured by disease-specific healthrelated quality-of-life questionnaires, is expected to increase in patients who also suffer from posttraumatic stress disorder (PTSD). Altered pain perception, sleep disorders, and fatigue may be associated with this comorbidity.

Methods: Severity of CRS was compared between a group of 28 patients with CRS and a group of 28 patients with CRS and concomitant PTSD using different disease-specific and generic instruments, such as visual analog scale (VAS), Short Form-36 test (SF-36), and Sino-Nasal Outcome Test-22 (SNOT 22).

Results: SNOT-22 test showed significantly higher CRS severity in patients with CRS and PTSD, compared to patients with CRS without PTSD.

Conclusion: Patients with less severe CRS, measured by objective outcome measures, due to the impact of comorbid PTSP, are classified as having severe rhinosinusitis, and are exposed to the risk of unnecessary diagnostic and therapeutic procedures. In patients with difficult-to-treat rhinosinusitis, diagnosis should be revised, and one item that should be evaluated is whether they suffer from PTSD.

Keywords: posttraumatic stress disorder, chronic rhinosinusitis, quality of life

\section{Introduction}

Chronic rhinosinusitis and posttraumatic stress disorder are diseases that strongly impact the quality of a person's life. ${ }^{1,2}$ The etiology of chronic rhinosinusitis is multifactorial, with different inflammatory mechanisms that can lead to the development the disease. The pathophysiology of the disease is intertwined, with numerous predisposing factors such as allergies, nonallergic hyperactivity, asthma, ciliary dyskinesia, and anatomic deformations. ${ }^{3}$

Diagnosis of rhinosinusitis has been made on the basis of recommendations found in the European Position on Rhinosinusitis and Nasal Polyps. The criteria are that the disease should last for more than 12 weeks with the existence of two or more symptoms, of which one has to be nasal blockage/congestion/obstruction or nasal/postnasal discharge. Other possible symptoms include facial pain or pressure and the reduction or loss of smell. The distinction between chronic rhinosinusitis without nasal polyps and chronic rhinosinusitis with nasal polyps is used to further point out differences in the pathophysiology and treatment of these two entities. $^{3}$

Posttraumatic stress disorder is characterized by persistent and nonremitting psychologically distressing reactions to a prior traumatic event. There are three 
main symptom clusters of posttraumatic stress disorder: (1) persistent re-experience of the event in a distressing way; (2) persistent avoidance of stimuli that remind one of the event; and (3) increased emotional arousal. ${ }^{4}$ It is assumed that a disorder in the synthesis of neurotransmitters in the hippocampus and amygdala contributes to the pathoanatomic background of the aforementioned symptoms. ${ }^{5}$

The majority of patients in Croatia who are diagnosed with posttraumatic stress disorder are diagnosed with combatrelated posttraumatic stress disorder, and the majority of these patients are men. Combat-related posttraumatic stress disorder is a clinically more severe disease than posttraumatic stress disorder caused by other forms of trauma. ${ }^{6}$ Given that both diseases are frequent in Croatia and other developed countries, the expected comorbidity of posttraumatic stress disorder and chronic rhinosinusitis is great.

Our hypothesis is that since patients who suffer from posttraumatic stress disorder and chronic rhinosinusitis simultaneously exhibit intense and widespread chronic pain, sleeping disorders, and fatigue, an additional deterioration of chronic rhinosinusitis symptoms occurs. The consequences of this are twofold: first, patients with the mild form of chronic rhinosinusitis are categorized into a group of patients with severe chronic rhinosinusitis, which exposes them to the risk of additional yet unnecessary therapies and diagnostic procedures; and second, with patients that do not react to the treatments, their diagnosis needs to be reviewed, and one of the differential diagnoses is posttraumatic stress disorder.

\section{Materials and methods}

\section{Patients}

The first group of patients was comprised of 28 males, whose chronic rhinosinusitis and posttraumatic stress disorder diagnosis had been confirmed. These patients had been hospitalized and treated in group therapy at the psychiatric ward of the County Hospital Virovitica in October 2010. They were all diagnosed with combat-related posttraumatic stress disorder. The second group was comprised of 28 male patients suffering from chronic rhinosinusitis alone, who were treated in the County Hospital Pakrac from June until October 2010. For all patients who were being treated for chronic rhinosinusitis and chronic rhinosinusitis/posttraumatic stress disorder, it is important to note that their diagnosis was based on the diagnostic criteria for chronic rhinosinusitis. This was confirmed by endoscopic results and computerized tomography scoring by Lund-Mackay, where the inclusion criterion was a score greater than 5 on this measure. Respondents that were included in the study did not suffer from other diseases. Both groups completed the Short Form-36 (SF-36), the Sino-Nasal Outcome Test-22 (SNOT-22), and visual analog scale (VAS) questionnaires.

\section{SF-36}

The SF-36 questionnaire measures patients' general health status and contains 36 questions that represent eight health concepts by grouping into subgroups, and each question is asked independently. Subgroups include physical functioning, role-physical, bodily pain, general health, vitality, social functioning, role-emotional, and mental health. One question that was observed independently relates to the change of the patient's health status in the year preceding the study, while the questions in the subgroups refer to the patient's status within the previous month. We used a Croatian version of the SF-36. Results are expressed as percentages, and the average total noted among the general Croatian population is $61.62 \%$. $^{7}$

\section{VAS}

The VAS questionnaire serves to evaluate the total severity of sinonasal symptoms, and the patient is asked to evaluate 18 questions, of which 12 are related to these symptoms, while the remaining six are related to general symptoms. The main question related to the patients' symptoms is: "What is the extent of problems related to the symptom at the moment?" Sinonasal symptoms include headaches, nose congestion, runny nose, secretion from the nose into the throat, feeling of fullness in the face, face pain, toothache, tearing, coughing, nose bleeds, and crusts in the nose. The remaining symptoms include general health, fatigue, fever, nausea, vomiting, and diarrhea-like stools. Patients identified problems according to the VAS, and possible scores ranged from 0 to $10 .^{8}$

\section{SNOT-22}

The SNOT-22 is a validated patient self-report measure of symptom severity and health-related quality of life in sinonasal conditions. The SNOT-22 test has 22 questions, and patients have to choose up to five important items that have affected their lives. Results of all questions are summed up, with higher results indicating greater problems; it should be noted that the maximum possible score is 110 points. Results for the general population fall around 30 points. ${ }^{9}$ We also observed the test by dividing it into two subscales. The first subscale covered physical symptoms that were 
related to "nasal problems;" these questions went as far as question 12. The second subscale covered health-related quality of life, including "emotional, fatigue, and sleep symptoms," and these included questions 13 to $22 .{ }^{10}$

\section{Results and analysis Statistical analysis}

We tested group differences with the Mann-Whitney $U$ test, and considered $P<0.05$ to be statistically significant. Data were tabulated in a Microsoft Excel (Microsoft Corporation, Redmond, WA) spreadsheet and imported into the Statistical Package for the Social Sciences (SPSS) version 10.0 (IBM Corporation, Armonk, NY).

All examined patients were males. The average age of respondents in the chronic rhinosinusitis group was $48.4 \pm 6.55$ years (range 38-61), while the average age of respondents in the posttraumatic stress disorder/chronic rhinosinusitis group was $45.3 \pm 4.89$ years (range $43-60$ ).

\section{VAS}

In the case of VAS test scoring, a lower score indicates a better result. The total VAS score was better among the chronic rhinosinusitis group at 3.8 points (range 1.7-6.2), and the score for the posttraumatic stress disorder/chronic rhinosinusitis group was 5.6 points (range 3.2-10). The difference was statistically significant $(P<0.05)$.

\section{SF-36}

In the case of the SF-36 test, the higher the result, the better the patient's general health. Results were better for the chronic rhinosinusitis group at a mean of $58.75 \%$, while for the posttraumatic stress disorder/chronic rhinosinusitis group, the result was a mean of $34.74 \%(P<0.05)$. Results were generally better among all of the eight test subgroups (Figure 1).

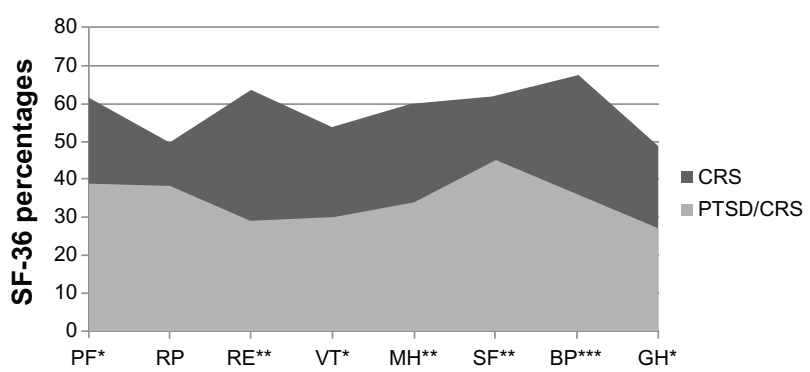

Figure I Short Form-36 (SF-36) test result percentages. Notes: $* P<0.05 ; * * P<0.001 ; * * * P<0.0001$

Abbreviations: PF, physical functioning; RP, role physical; RE, role emotional; $\mathrm{VT}$, vitality; $\mathrm{MH}$, mental health; SF, social functioning; BP, bodily pain; $\mathrm{GH}$, general health; CRS, chronic rhinosinusitis; PTSD, posttraumatic stress disorder.

\section{SNOT-22}

In the case of SNOT-22 testing, a lower score is indicative of a better result. Better results were found among the chronic rhinosinusitis group. The mean SNOT-22 sum score was 48.80 in the chronic rhinosinusitis group (range 44-51) and 58.33 in the posttraumatic stress disorder/chronic rhinosinusitis group (range 37-84) $(P<0.05)$ (Figure 2).

In the chronic rhinosinusitis group, the two most important symptoms are runny nose and nasal obstructions, and in the chronic rhinosinusitis/posttraumatic stress disorder group, the most important symptoms are the difficulties experienced when falling asleep and waking up at night.

By dividing the SNOT-22 test after question 12, we created two subscales: the first being "emotional, fatigue, and sleep symptoms," and the second being "nasal problems." We compared what percentage of patients chose "five important terms which affected their lives" from the two aforementioned groups, and we observed that $38 \%$ of patients in the chronic rhinosinusitis/posttraumatic stress disorder group chose items that were solely related to "emotional, fatigue, and sleep symptoms," $12 \%$ chose items related to "nasal problems" alone, and 50\% chose both or none. In contrast, $39 \%$ of patients in the chronic rhinosinusitis group chose items related to "nasal problems" alone, 13\% chose items related to "emotional, fatigue, and sleep symptoms" alone, and $48 \%$ chose both or none (Figure 3 ).

\section{Discussion}

Chronic rhinosinusitis has adverse effects on quality of life, just like diabetes or heart disease. ${ }^{11}$ In the USA, $15 \%$ of the population suffers from this condition, and it is responsible for 13 million visits to a physician and 2 million hospitalizations per year. ${ }^{12,13}$ As per research that investigated 361 Gulf War veterans upon their return home, the most

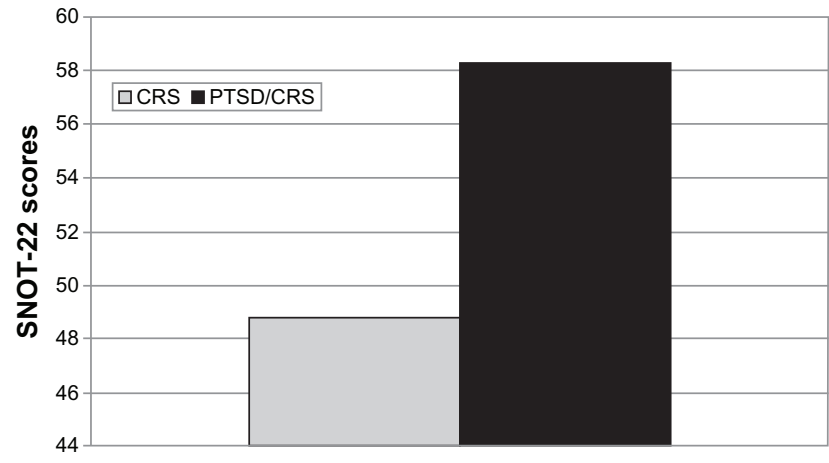

Figure 2 Sino-Nasal Outcome Test-22 (SNOT-22) results. Note: $* P<0.05$.

Abbreviations: CRS, chronic rhinosinusitis; PTSD, posttraumatic stress disorder. 


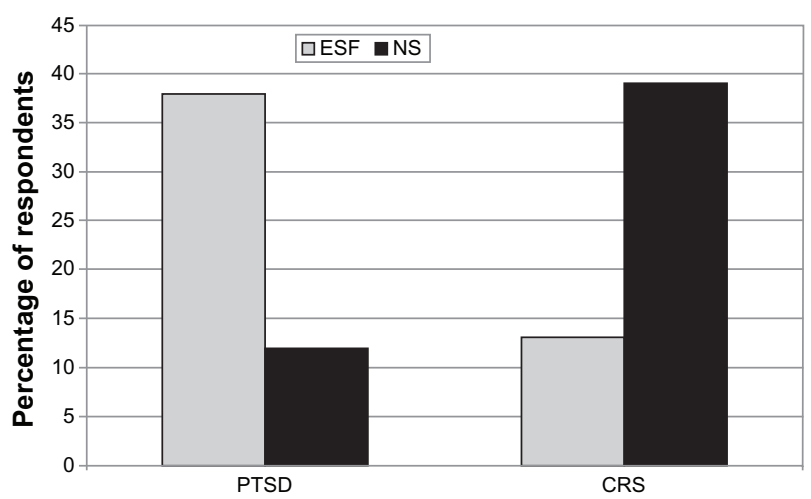

Figure 3 Percentage of respondents from each of the two subgroups that chose either "emotional, fatigue, and sleep symptoms," or "nasal symptoms" from the "five important items which affected their lives" on the SNOT-22 test.

Abbreviations: SNOT-22, Sino-Nasal Outcome Test-22; ESF, emotion, sleep, fatigue; NS, nasal symptoms; PTSD, posttraumatic stress disorder; CRS, chronic rhinosinusitis.

frequent diagnosis in head and neck diseases was chronic rhinosinusitis..$^{14}$ Posttraumatic stress disorder is the fourth most frequent psychiatric diagnosis in the USA, affecting $10 \%$ of males and $18 \%$ of females. ${ }^{15}$ From the aforementioned data, it is apparent that the expected comorbidity of chronic rhinosinusitis and posttraumatic stress disorder is large.

Chronic rhinosinusitis is a disease that is diagnosed on the basis of subjective symptoms, which means that only anamnesis and clinical examination are needed as diagnostics. An objective method like computed tomography is widely accepted, but it is insufficient in monitoring a patient's condition because it correlates poorly with subjective symptoms. ${ }^{16}$ The scoring of computed tomography results by Lund-Mackay provides the best correlation of computed tomography results and subjective sinonasal symptoms; however, this correlation is lost with nonnasal sinonasal symptoms. ${ }^{17}$ Objectivization is also possible according to endoscopic grading, which is widely accepted, but this is dependent on the physician's subjective assessment. Due to these weaknesses in these objective methods, evaluation by subjective tests provides the most valuable information about the course of the disease, therapy success, and the effects of the disease on each patient's quality of life. ${ }^{18}$

Confusion in evaluating a patient's condition is caused by the division of symptoms as either minor or major. Namely, the term "minor" speaks to the sensitivity and specificity of a certain symptom as a diagnostic criterion, and does not address its prevalence or severity. Contrary to popular opinion, minor symptoms of headache and facial pressure are not the leading symptoms of rhinosinusitis; rather, fatigue prevails at a rate of $84 \% .{ }^{19}$ With the detailed diagnosis and treatment of patients with facial pain as the obstinate symptom, we most often remove chronic rhinosinusitis as the cause of pain, and reach a neurological diagnosis. ${ }^{20,21}$ Only recently was posttraumatic stress disorder's effect on chronic orofacial pain identified. It has also been identified that males with posttraumatic stress disorder are not the only ones who exhibit intense chronic pain; their spouses also report experiencing pain, often in the form of headaches..$^{22,23}$

With patients who report fatigue, it should be taken into consideration that $46 \%$ of patients with chronic fatigue syndrome suffer from nonallergic chronic rhinosinusitis, and that $26 \%$ of patients with chronic rhinosinusitis suffer from chronic fatigue syndrome. ${ }^{24}$ The correlation of chronic rhinosinusitis and chronic fatigue syndrome is well researched. The deterioration of symptoms in comorbid conditions is interpreted as having systematic nociceptive dysfunction that is not conditioned by local inflammatory response in the sinonasal area; rather, this pain arises from the central nervous system and the opioid system of the posterior horns of the spinal cord. ${ }^{25}$

Posttraumatic stress disorder and chronic fatigue syndrome symptoms are similar to those noted in many "wartime" syndromes (soldier's heart, shellshock, Gulf War syndrome) and "peacetime" syndromes (fibromyalgia, somatization disorders, and multiple chemical sensitivities), and this correlation is the topic of much research. ${ }^{26}$ The similarities of the pathophysiological changes of posttraumatic stress disorders and chronic fatigue syndrome that are taking place in the central nervous system (particularly in the amygdala and hypothalamus), point to the great potential of future research about the correlation of posttraumatic stress disorder and chronic rhinosinusitis, among other things. This is because posttraumatic stress disorder is much more frequent in the general population than chronic fatigue syndrome. ${ }^{27-29}$ All three questionnaires that we used in our study have shown that posttraumatic stress disorder deteriorates the symptoms of chronic rhinosinusitis, which then classifies patients into the severe group. The VAS that we used was adjusted to assess a patient's current condition. Results have proven to us that both groups are currently in the active phase of the disease.

Studies that have applied the SF-36 test to compare the quality of life of patients with chronic rhinosinusitis and of patients with other chronic diseases like lumbago, chronic heart failure, and obstructive pulmonary diseases have shown that patients with chronic rhinosinusitis have poorer social functioning and higher pain sensitivity than patients with the other conditions. ${ }^{1,11}$ The chronic rhinosinusitis group from our research reported experiencing lower qualities of life, 
which generated poorer total test results $(58.75 \%)$; this was compared to the quality of life of the general population in Croatia, which was considerably higher $(61.62 \%))^{7}$

The SNOT-22 test is a test that consolidates sinonasal symptoms and some general health symptoms. The SNOT-22 test is used for the purpose of tracking the success in treating chronic rhinosinusitis. What is noticeable is the tendency that nasal problems contribute to the deterioration of test scores in the group, while in the chronic rhinosinusitis/posttraumatic stress disorder group, test scores are affected by emotional problems, fatigue, and sleep (Figure 3). Significantly poorer results among the chronic rhinosinusitis/posttraumatic stress disorder group point to a need for cooperation between the psychiatrist and otolaryngologist when treating chronic rhinosinusitis because the deterioration of symptoms of rhinosinusitis, particularly without objective indicators, can be caused by the deterioration of posttraumatic stress disorder. By keeping this fact in mind, we can not only save the patient from additional pharmacological treatments, especially to reduce the pain, but we can also save patients from unnecessary surgeries. Operated patients with chronic rhinosinusitis/ posttraumatic stress disorder can have poor or no postoperative recovery, which is frustrating for the patient and the surgeon. The problem can be looked at from another angle: in the case of patients with obdurate chronic rhinosinusitis symptomatology and/or poor postoperative recovery, the diagnosis needs to be reviewed and posttraumatic stress disorder must be taken into consideration as a potential comorbidity.

\section{Conclusion}

Higher chronic pain sensitivity, sleeping disorders, and tiredness in chronic rhinosinusitis/posttraumatic stress disorder patients aggravate the scores of chronic rhinosinusitis; therefore, these patients are classified as belonging to a group with severe rhinosinusitis. Patients with chronic rhinosinusitis that do not show signs of improvement with therapy may have comorbid posttraumatic stress disorder.

\section{Disclosure}

The authors report no conflicts of interest in this work.

\section{References}

1. van Agthoven M, Fokkens WJ, van de Merwe JP, Marijke van Bolhuis E, Uyl-de Groot CA, Busschbach JJ. Quality of life of patients with refractory chronic rhinosinusitis: effects of filgrastim treatment. Am J Rhinol. 2001;15(4):231-237.

2. McWilliams LA, Cox BJ, Asmundson GJ. Symptom structure of posttraumatic stress disorder in a nationally representative sample. J Anxiety Disord. 2005;19(6):626-641.
3. Fokkens W, Lund V, Mullol J; European Position Paper on Rhinosinusitis and Nasal Polyps Group. EP3OS 2007: European position paper on rhinosinusitis and nasal polyps 2007. A summary for otorhinolaryngologists. Rhinology. 2007;45(2):97-101.

4. Hinton DE, Lewis-Fernández R. The cross-cultural validity of posttraumatic stress disorder: implications for DSM-5. Depress Anxiety. 2011;28(9):783-801.

5. Schmahl C, Berne K, Krause A, et al. Hippocampus and amygdala volumes in patients with borderline personality disorder with or without posttraumatic stress disorder. J Psychiatry Neurosci. 2009;34(4):289-295.

6. Arbanas G. Patients with combat-related and war-related posttraumatic stress disorder 10 years after diagnosis. Croat Med J. 2010;51(3): 209-214.

7. Maslić Sersić D, Vuletić G. Psychometric evaluation and establishing norms of Croatian SF-36 health survey: framework for subjective health research. Croat Med J. 2006;47(1):95-102.

8. Lim M, Lew-Gor S, Darby Y, Brookes N, Scadding G, Lund VJ. The relationship between subjective assessment instruments in chronic rhinosinusitis. Rhinology. 2007;45(2):144-147.

9. Hopkins C, Gillett S, Slack R, Lund VJ, Browne JP. Psychometric validity of the 22-item Sinonasal Outcome Test. Clin Otolaryngol. 2009;34(5):447-454.

10. Browne JP, Hopkins C, Slack R, Cano SJ. The Sino-Nasal Outcome Test (SNOT): can we make it more clinically meaningful? Otolaryngol Head Neck Surg. 2007;136(5):736-741.

11. Glikich RE, Metson R. The health impact of chronic sinusitis in patients seeking otolaryngologic care. Otolaryngol Head Neck Surg. 1995; 113(1):104-109.

12. Blackwell DL, Collins JG, Coles R. Summary health statistics for US adults: National Health Interview Survey, 1997. Vital Health Stat 10. 2002;205:1-109.

13. Schappert SM, Rechtsteiner EA. Ambulatory medical care utilization estimates for 2006. Natl Health Stat Report. 2008;8:1-29.

14. Ladich ER, Lewin-Smith MR, Specht CS, Moroz AL, Kalasinsky VF, Mullick FG. A histopathological study of head and neck specimens from a cohort of Persian Gulf War military veterans. Mil Med. 2002;167(10): 864-867.

15. Breslau N, Kessler RC, Chilcoat HD, Schultz LR, Davis GC, Andreski P. Trauma and posttraumatic stress disorder in the community: the 1996 Detroit Area Survey of Trauma. Arch Gen Psychiatry. 1998;55(7): 626-632.

16. Bhattacharyya N. A comparison of symptom scores and radiographic staging systems in chronic rhinosinusitis. Am J Rhinol. 2005;19(2): $175-179$.

17. Lund VJ, Kennedy DW. Staging for rhinosinusitis. Otolaryngol Head Neck Surg. 1997;117(3 Pt 2):S35-S40.

18. Chester AC, Sindwani R. Symptom outcomes in endoscopic sinus surgery: a systematic review of measurement methods. Laryngoscope. 2007;117(12):2239-2243.

19. Bhattacharyya $\mathrm{N}$. The economic burden and symptom manifestations of chronic rhinosinusitis. Am J Rhinol. 2003;17(1):27-32.

20. Paulson EP, Graham SM. Neurologic diagnosis and treatment in patients with computed tomography and nasal endoscopy negative facial pain. Laryngoscope. 2004;114(11):1992-1996.

21. West B, Jones NS. Endoscopy-negative, computed tomographynegative facial pain in a nasal clinic. Laryngoscope. 2001;111(4 Pt 1): 581-586.

22. Cyders MA, Burris JL, Carlson CR. Disaggregating the relationship between posttraumatic stress disorder symptom clusters and chronic orofacial pain: implications for the prediction of health outcomes with PTSD symptom clusters. Ann Behav Med. 2011;41(1):1-12.

23. Koić E, Francišković T, Mužinić-Masle L, Đorđević V, Vondraček S, Pripić J. Chronic pain and secondary traumatization in wives of Croatian war veterans treated for posttraumatic stress disorder. Acta Clin Croat. 2002;41:295-306.

24. Baraniuk JN, Zheng Y. Relationships among rhinitis, fibromyalgia, and chronic fatigue. Allergy Asthma Proc. 2010;31(3):169-178. 
25. Baraniuk JN, Petrie KN, Le U, et al. Neuropathology in rhinosinusitis. Am J Respir Crit Care Med. 2005;171(1):5-11.

26. Eglinton R, Chung MC. The relationship between posttraumatic stress disorder, illness cognition, defence styles, fatigue severity and psychological well-being in chronic fatigue syndrome. Psychiatry Res. 2011; 188(2):245-252.

27. Kroes MC, Rugg MD, Whalley MG, Brewin CR. Structural brain abnormalities common to posttraumatic stress disorder and depression. J Psychiatry Neurosci. 2011;36(4):256-265.
28. Okada T, Tanaka M, Kuratsune H, Watanabe Y, Sadato N. Mechanisms underlying fatigue: a voxel-based morphometric study of chronic fatigue syndrome. BMC Neurol. 2004;4(1):14.

29. Gupta A. Unconscious amygdalar fear conditioning in a subset of chronic fatigue syndrome patients. Med Hypotheses. 2002;9(6):727-735.

Patient Preference and Adherence

Dovepress

\section{Publish your work in this journal}

Patient Preference and Adherence is an international, peer-reviewed, open access journal focusing on the growing importance of patient preference and adherence throughout the therapeutic continuum. Patient satisfaction, acceptability, quality of life, compliance, persistence and their role in developing new therapeutic modalities and compounds to optimize clinical outcomes for existing disease states are major areas of interest. This journal has been accepted for indexing on PubMed Central. The manuscript management system is completely online and includes a very quick and fair peer-review system. Visit http://www.dovepress.com/ testimonials.php to read real quotes from published authors.

Submit your manuscript here: http://www.dovepress.com/patient-preference-and-adherence-journal 